\title{
A Comparison of Totally Laparoscopic Pylorus Preserving Gastrectomy and Laparoscopy-Assisted Pylorus Preserving Gastrectomy for Early Gastric Cancer
}

\author{
Won Ho Han, M.D. ${ }^{1}$, Bang Wool Eom, M.D., Ph.D. ${ }^{1}$, Hong Man Yoon, M.S., M.D. ${ }^{1}$, Keun Won Ryu, M.D., Ph.D. ${ }^{1}$, \\ Deok Hee Kim, R.N.', Young-Woo Kim, M.D., Ph.D. ${ }^{1,2}$ \\ ${ }^{1}$ Center for Gastric Cancer, National Cancer Center, ${ }^{2}$ Department of Cancer Control and Population Health, National Cancer Center Graduate School of \\ Cancer Science and Policy, Goyang, Korea
}

Purpose: Pylorus-preserving gastrectomy (PPG) is known to have both nutritional and functional advantages over distal gastrectomy for the treatment of early gastric cancer. Although laparoscopic surgery is a popular choice, intracorporeal anastomosis is a newly developed technique that is gaining popularity. This study aimed to determine any differences in the oncological, surgical, and functional outcomes of intracorporeal and extracorporeal anastomosis after PPG.

Methods: A retrospective analysis was performed on 90 patients for cT1N0 gastric cancer who underwent laparoscopic pylorus preserving gastrectomy from January 2015 to June 2017 at the OOO, Korea; 38 patients underwent intracorporeal (TLPPG) and 52 underwent extracorporeal (LAPPG) anastomosis. The postoperative oncological, surgical, and functional outcomes were compared between the two groups. In order to compare the outcomes in obese patients, the postoperative and functional outcomes in patients with a BMI of $\geq 25$, and in those with abdominal wall thickness measuring $\geq 28 \mathrm{~mm}$, were evaluated.

Results: The TLPPG group showed a significantly reduced wound size $(4 \mathrm{~cm}(3 \sim 4)$ vs $5 \mathrm{~cm}(5 \sim 6)$, $p<0.001)$ and had fewer wound complaints than the LAPPG group (0.0\% vs $15.4 \%, p=0.01)$. Postoperative complications were not significantly different between the two groups. In the BMI $\geq 25$ subgroup, the first flatus time after operation was shorter in the TLPPG group (2.9 \pm 0.5 vs $3.5 \pm 0.8$ days, $p=0.04$ ).

Conclusion: The study demonstrates that both TLPPG and LAPPG are safe and feasible, and that there is a potential benefit for obese patients.

Keywords: Gastric cancer, Surgery, Anastomosis, Laparoscopy, Pylorus preserving gastrectomy

This is an Open Access article distributed under the terms of the Creative Commons Attribution Non-Commercial License (http:// creativecommons.org/licenses/by-nc/4.0/) which permits unrestricted non-commercial use, distribution, and reproduction in any medium, provided the original work is properly cited.
Received May 13, 2019

Revised July 4, 2019

Accepted July 10, 2019

Corresponding author

Young-Woo Kim

Department of Cancer Control and

Population Health, National Cancer

Center Graduate School of Cancer

Science and Policy, 323 Ilsan-ro,

Ilsandong-gu, Goyang 10408, Korea

Tel: +82-31-920-1635

Fax: +82-31-920-1895

E-mail: gskim@ncc.re.kr

ORCID:

https://orcid.org/0000-0002-1559-9672

\section{INTRODUCTION}

Laparoscopic gastrectomy has become the standard treatment used in the treatment of early gastric cancer, and many laparoscopic intracorporeal anastomosis methods have been developed. ${ }^{1-3}$ In gastroduodenostomy after distal gastrectomy, several studies have shown that delta-shaped intracorporeal anastomosis is both safe and feasible., ${ }^{4,5}$ Compared with extracorporeal anastomosis, the incision is smaller, cosmetically superior and less painful, which can reduce the use of anal- 
gesics and wound infections, and improve the operation field, especially in obese patients. ${ }^{6}$

Gastro-gastrostomy anastomosis after pylorus preserving gastrectomy has been reported to have various advantages over extracorporeal anastomosis. ${ }^{7-9}$ However, pylorus preserving gastrectomy has a disadvantage in that it is more difficult to use a stapler compared with reconstruction after distal gastrectomy; therefore, there are fewer studies on intracorporeal reconstruction. A number of studies have reported the short-term operative outcome for totally laparoscopic pylorus preserving gastrectomy (TLPPG) using a linear stapler. ${ }^{10-12}$ However, these studies have involved only a small number of patients and have not compared the outcomes to existing laparoscopic assisted pylorus preserving gastrectomy (LAPPG). It is also important to determine whether there is an advantage or disadvantage to performing a particular type of anastomosis. In this study, we compared the safety and feasibility of LAPPG and TLPPG.

\section{MATERIALS AND METHODS}

A retrospective case control study was conducted on 90 patients who underwent laparoscopic pylorus preserving gastrectomy from January 2015 to June 2017 at the National Cancer Center, Korea. All patients were diagnosed with cT1N0 on preoperative endoscopy and abdominal CT scan.

Laparoscopic assisted pylorus preserving gastrectomy, with a hand-sewn suture, was performed in 52 patients, and TLPPG, with a linear stapler, was performed in 38 patients. In LAPPG, the epigastric incision was made transversely for gastro-gastrostomy. In TLPPG, gastro-gastrostomy anastomosis was performed as side-to-side gastro-gastrostomy using a linear stapler (piercing method). ${ }^{11}$ In both group, $12 \mathrm{~mm}$ balloon trocar was inserted in the umbilicus area and two $12 \mathrm{~mm}$ trocars (left lower quadrant, right lower quadrant site) $5 \mathrm{~mm}$ trocars (left upper quadrant, right upper quadrant site) were inserted. The main wound of TLPPG was made by extending the umbilical port incision. In the LAPPG, an additional epigastric incision was made for anastomosis. The main wound size was measured after surgery by the surgeon.

The oncologic outcome (retrieved lymph node count, proximal margin, and distal margin), intraoperative outcome (operating time and bleeding volume), and postoperative outcome (postoperative 1 and 3 day pain score, additional IV pain relief, first flatus passage after operation, hospital days after operation, presence of reflux symptoms, main wound size, presence of dumping symptoms, and surgical wound complaints) were investigated. The postoperative complications were evaluated according to the Clavien-Dindo classification.

We compared the postoperative outcomes and complications between the two groups in the obese patients with BMI $\geq 25^{13}$ and those with abdominal wall thickness $>28 \mathrm{~mm}$ as abdominal obesity.

All analyses were performed using SAS ${ }^{\circledast}$ version 9.1.3 for Windows ${ }^{\circledR}$ (SAS Institute, Cary, North Carolina, USA). Categorical variables were compared using the Pearson $\chi^{2}$ test or Fisher's exact test, and continuous variables were compared using Student's t-test. All values are expressed as mean \pm standard error of the mean. Statistical significance was set at $p<0.05$.

\section{RESULTS}

\section{Patients demographics}

The demographic and clinicopathological characteristics of patients with LAPPG and TLPPG were not significantly different between the two groups. Although all patients were diagnosed with early gastric cancer (cT1) preoperatively, $2 \mathrm{pa}^{-}$ tients in the TLPPG group and 4 patients in the LAPPG group were revealed to have greater than pStage II (Table 1).

\section{Surgical outcome}

In terms of postoperative outcomes, the TLPPG group showed a significantly reduced wound size $(4 \mathrm{~cm}(3 \sim 4)$ vs 5 $\mathrm{cm}(5 \sim 6), p<0.001)$. Eight patients in the LAPPG group reported surgical wound complaints, while there were no wound complaints in the TLPPG patients. The TLPPG group had less reflux symptoms at the outpatient clinic than the LAPPG group, but the difference was not significant (28.9\% vs $36.5 \%$, $p=0.45$ ). One patient in the LAPPG group reported a dumping symptom (Table 2). There were no differences between the retrieved lymph nodes and the distal and proximal margins when the short-term operative outcomes were compared between groups. The operating time and the mean amount of bleeding during operation were also not significantly different.

\section{Postoperative complications}

None of the measured postoperative complications or severe complications of Clavien-Dindo classification (more than grade II) were significantly different between the two groups ( $p=0.74$ and $p=0.64$, respectively). There were two complications relating to anastomosis leakage in both groups; endoscopic clipping was performed for anastomosis site leakage. Delayed gastric emptying occurred in five cases in the LAPPG group, and one case was diagnosed as delayed gastric emptying in the TLPPG group. Five patients were improved after NPO and fluid therapy; however endoscopic Botox injection 
Table 1. Preoperative demographic and clinicopathological factors

\begin{tabular}{lccc}
\hline & TLPPG $(\mathbf{n}=38)$ & LAPPG $(\mathbf{n}=52)$ & $p$ value \\
\hline Age & $58.6 \pm 10.7$ & $56.8 \pm 10.7$ & 0.71 \\
Sex & & & 0.20 \\
Male & $16(42.1 \%)$ & $29(55.8 \%)$ & \\
Female & $22(57.9 \%)$ & $23(44.2 \%)$ & \\
BMI (kg/m $\left.{ }^{2}\right)$ & $23.7 \pm 2.9$ & $23.6 \pm 2.8$ & 0.81 \\
Abdominal wall & $27.6 \pm 5.8$ & $29.2 \pm 7.3$ & 0.28 \\
thickness (mm) & & & \\
ASA score* & & & 0.10 \\
1 & $22(57.9 \%)$ & $20(38.5 \%)$ & \\
2 & $15(39.5 \%)$ & $31(59.6 \%)$ & \\
3 or more & $1(2.6 \%)$ & $1(1.9 \%)$ & \\
pT category & & & 0.86 \\
1 & $34(89.5 \%)$ & $47(90.4 \%)$ & \\
2 & $3(7.9 \%)$ & $3(5.8 \%)$ & \\
3 & $1(2.6 \%)$ & $2(3.8 \%)$ & \\
pN category & & & 0.65 \\
0 & $34(89.5 \%)$ & $46(88.5 \%)$ & \\
1 & $2(5.3 \%)$ & $5(9.6 \%)$ & \\
2 & $2(5.3 \%)$ & $1(1.9 \%)$ & \\
pStage & & & 0.70 \\
I & $36(94.7 \%)$ & $48(92.3 \%)$ & \\
II & $0(0.0 \%)$ & $4(7.7 \%)$ & \\
III & $2(5.3 \%)$ & $0(0.0 \%)$ & \\
\hline & & & \\
\hline
\end{tabular}

${ }^{*} A S A=$ American society of anesthesiologists.

treatment was performed in one patient in the LAPPG group due to a lack of effect with conservative therapy (Table 3).

\section{Subgroup analysis}

There was no significant difference in postoperative complications between the patients with BMI $\geq 25$ and above. The hospital days after operation were shorter in the TLPPG group but not statistically significant ( $7.5 \pm 1.1$ vs $9.5 \pm 2.5, p=0.25$ ). The first flatus time after the operation was shorter in the TLPPG group ( $2.9 \pm 0.5$ vs $3.5 \pm 0.8, p=0.04$ ) (Table 4).

When only comparing patients with an abdominal wall thickness $>28 \mathrm{~mm}$, the postoperative complications $(15.0 \%$ vs $25.8 \%, p=0.49)$ and the reflux symptoms after operation $(25.0 \%$ vs $38.7 \%, p=0.37$ ) were less in the TLPPG group, but not statistically significant (Table 5).
Table 2. Short-term operative outcomes

\begin{tabular}{lccc}
\hline & TLPPG $(\mathbf{n}=38)$ & LAPPG $(\mathbf{n}=52)$ & $p$ value \\
\hline Oncologic outcome & & & \\
Retrieved LN & $35.0 \pm 11.7$ & $32.3 \pm 10.4$ & 0.25 \\
Distal margin (cm) & $2.9 \pm 2.3$ & $3.4 \pm 3.1$ & 0.34 \\
Proximal margin (cm) & $2.5 \pm 1.5$ & $2.1 \pm 1.8$ & 0.23 \\
Intraoperative outcome & & & \\
Operating time (min) & $216.7 \pm 41.1$ & $197.8 \pm 43.3$ & 0.40 \\
Estimated blood loss & $25(20 \sim 50)$ & $22.5(20 \sim 70)$ & 0.43 \\
Postoperative outcome & & & \\
NRS* on post- & $4.5 \pm 1.3$ & $4.8 \pm 1.7$ & 0.35 \\
operative 1 day & & & \\
NRS* on post- & $3.5 \pm 0.5$ & $3.4 \pm 1.0$ & 0.29 \\
operative 3 day & & & \\
Main wound & $4(3 \sim 4)$ & $5(5 \sim 6)$ & $<0.001$ \\
size** (cm) & & & \\
Wound complaint & $0(0 \%)$ & $8(15.4 \%)$ & 0.01 \\
First flatus time & $3.2 \pm 0.7$ & $3.4 \pm 0.7$ & 0.37 \\
Hospital day (days) & $7.7 \pm 2.3$ & $7.3 \pm 2.0$ & 0.79 \\
Reflux symptoms & $11(28.9 \%)$ & $19(36.5 \%)$ & 0.45 \\
Dumping symptoms & 0 & $1(2.0 \%)$ & 1.00 \\
\hline
\end{tabular}

${ }^{*}$ Numerical rating scale (pain scale). ${ }^{* *}$ The main wound of TLPPG is the size of the incision that extends the umbilical port site, and LAPPG is the additional epigastric incision.

Table 3. Postoperative complications

\begin{tabular}{lccc}
\hline & $\begin{array}{c}\text { TLPPG } \\
(\mathbf{n}=38)\end{array}$ & $\begin{array}{c}\text { LAPPG } \\
(\mathbf{n}=52)\end{array}$ & $p$ value \\
\hline All & $7(18.4 \%)$ & $11(21.2 \%)$ & 0.74 \\
\hline Anastomosis leakage & 2 & 2 & \\
Delayed emptying & 2 & 5 & \\
Anastomosis stenosis & 2 & 1 & \\
Complicated fluid collection & 1 & 2 & \\
Wound infection & 0 & 1 & \\
C-D* III \& IV & $4(10.5 \%)$ & $4(7.7 \%)$ & 0.64 \\
Anastomosis leakage & 2 & 2 & \\
Anastomosis stenosis & 1 & 1 & \\
Delayed emptying & 0 & 1 & \\
Fluid collection & 1 & 0 & \\
\hline
\end{tabular}

${ }^{*}$ Clavien-Dindo classification. 
The postoperative pain score was slightly lower in the TLPPG group, but not statistically significant in subgroup analyses (Fig. 1).

\section{DISCUSSION}

The aim of the present study was to compare the short-term outcomes of TLPPG and LAPPG. In terms of the surgical wound, intracorporeal anastomosis is superior to extracorporeal anastomosis. In addition, TLPPG showed better functional outcome in obese patients.

Totally laparoscopic pylorus preserving gastrectomy is an intracorporeal anastomosis whereby, theoretically, tension free anastomosis is possible and it is possible to perform the anastomosis with a small incision extension. ${ }^{11}$ It is suggested that

Table 4. Postoperative outcome in obese patients (BMI $>25)$

\begin{tabular}{lccc} 
& $\begin{array}{c}\text { TLPPG } \\
(\mathbf{n}=14)\end{array}$ & $\begin{array}{c}\text { LAPPG } \\
(\mathbf{n}=14)\end{array}$ & $p$ value \\
\hline Postoperative outcome & & & \\
First flatus time & $2.9 \pm 0.5$ & $3.5 \pm 0.8$ & 0.04 \\
Hospital day (days) & $7.5 \pm 1.1$ & $9.5 \pm 2.5$ & 0.25 \\
Reflux symptoms & $4(28.6 \%)$ & $7(50.0 \%)$ & 0.44 \\
Main wound size $(\mathrm{cm})$ & $4(4 \sim 5)$ & $5(5 \sim 6)$ & $<0.001$ \\
Wound complaint & $0(0.0 \%)$ & $1(7.1 \%)$ & 1.00 \\
Postoperative complications & $5(35.7 \%)$ & $3(21.4 \%)$ & 0.67 \\
Severe complications & $2(14.3 \%)$ & $3(21.4 \%)$ & 1.00 \\
\hline
\end{tabular}

*Clavien-Dindo classification grade III and IV.

A

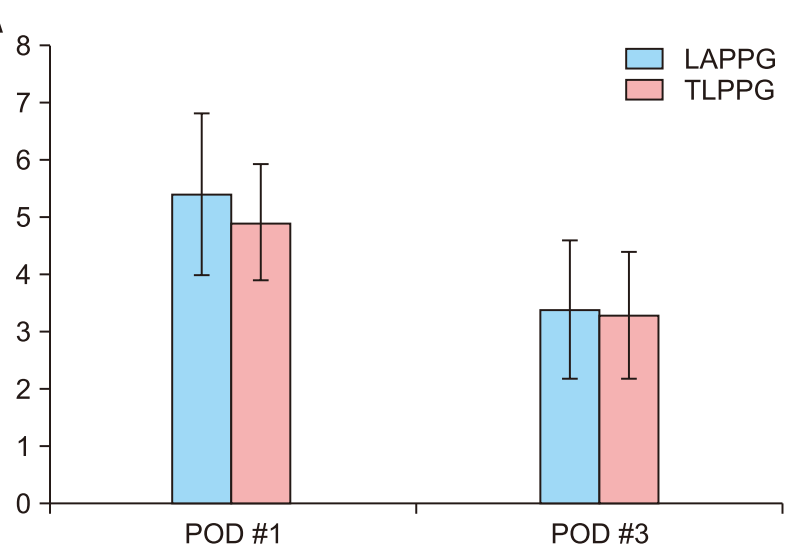

TLPPG reduces complications and postoperative pain compared to LAPPG. Totally laparoscopic pylorus preserving gastrectomy is feasible in all patients, but could provide a better working space and vision in obese patients and is also helpful since there is no need to extend the wound. ${ }^{14}$

In terms of postoperative outcome, the only wound complaint in the LAPPG group was because of the wound extension. The LAPPG requires an incision in the upper midline approximately $5 \mathrm{~cm}$ in size. However, the lack of wound complaint in the TLPPG group can be explained by the fact that it is possible to extend only $3 \sim 4 \mathrm{~cm}$ from the umbilical port, so there is less wound complaint. In obese patients, LAPPG may require a wound extension to maintain the visual field; however, TLPPG does not require this wound extension. In

Table 5. Postoperative outcomes in patients with abdominal wall thickness $>28 \mathrm{~mm}$

\begin{tabular}{lccc}
\hline & $\begin{array}{c}\text { TLPPG } \\
(\mathbf{n}=20)\end{array}$ & $\begin{array}{c}\text { LAPPG } \\
(\mathbf{n}=31)\end{array}$ & $p$ value \\
\hline Postoperative outcome & & & \\
First flatus time & $3.2 \pm 0.7$ & $3.3 \pm 0.8$ & 0.85 \\
Hospital day (days) & $7.8 \pm 3.0$ & $7.2 \pm 1.0$ & 0.25 \\
Reflux symptoms & $5(25.0 \%)$ & $12(38.7 \%)$ & 0.37 \\
Main wound size & $4(4 \sim 5)$ & $5(5 \sim 6)$ & $<0.001$ \\
Wound complaint & $0(0.0 \%)$ & $3(9.7 \%)$ & 0.27 \\
Postoperative complications & $3(15.0 \%)$ & $8(25.8 \%)$ & 0.49 \\
Severe complications & $1(5.0 \%)$ & $3(9.7 \%)$ & 0.54 \\
\hline
\end{tabular}

*Clavien-Dindo classification grade III and IV.

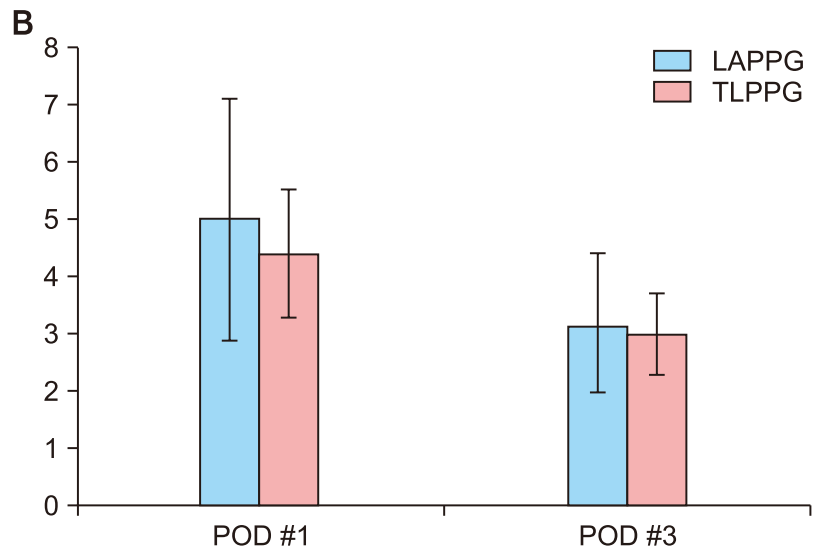

Fig. 1. Comparison of postoperative numerical pain rating scale. (A) Among the patients above BMI 25 , numerical pain rating scale were lower in the TLPPG group but not statistically significant (5.4 \pm 1.4 vs $4.9 \pm 1.0 p=0.23$ in postoperative 1 day, $3.4 \pm 1.2$ vs $3.3 \pm 1.1 p=0.41$ in postoperative 3 day). (B) Among the patients who have abdominal wall thickness greater than $28 \mathrm{~mm}$, numerical pain rating scale were lower in the TLPPG group but not statistically significant $(5.0 \pm 2.1$ vs $4.4 \pm 1.1 p=0.08$ in postoperative 1 day, $3.2 \pm 1.2$ vs $3.0 \pm 0.7 p=0.56$ in postoperative 3 day). 
the present study, there was no significant difference in wound complications between intracorporeal and extracorporeal anastomosis; this is in agreement with previous studies. ${ }^{4,5,15}$

Laparoscopic assisted pylorus preserving gastrectomy has a limited working space and narrow field of view during anastomosis. However, TLPPG can avoid this limitation of field and can be particularly helpful in obese patients. Theoretically, the working space and visual field limitations are less than with LAPPG, which can reduce tension during anastomosis. Tension-free anastomosis is possible and damage of surrounding structures can be avoided. A previous study comparing TLDG and LADG also reported the same advantages of intracorporeal anastomosis. ${ }^{16,17}$ In the present study, obese patients with a BMI $\geq 25$ and an abdominal wall thickness $>28 \mathrm{~mm}$ had a shorter first flatus time after the operation in TLPPG group. This result suggests that TLPPG is more effective for bowel recovery after surgery.

Unlike LAPPG, TLPPG could not confirm lesion or clipping site during resection. Therefore, there is a possibility that the resection margin is short, or that the residual tumor is in the margin. In this study, a frozen biopsy was performed in all cases in order to ensure a negative margin. In previous studies, there was a statistically significant difference in the resection margin between the two groups; however, none of these studies reported residual tumor in the margin. ${ }^{5,6,18,19}$

This study has a limitation in that it is an explorative study of a small case series compared to a control group. As yet, there is no long-term data for the oncological outcome, longterm complications, and quality of life benefit. Furthermore, symptoms were measured subjectively based on the patient's complaint; however, these symptoms did not have objective indicators.

Both TLPPG and LAPPG are safe and feasible, and intracorporeal anastomosis after pylorus preserving gastrectomy has potential benefits and warrants further development.

\section{ORCID}

Won Ho Han, https://orcid.org/0000-0002-7835-7603

Bang Wool Eom, https://orcid.org/0000-0002-0332-2051

Hong Man Yoon, https://orcid.org/0000-0002-6218-7080

Keun Won Ryu, https://orcid.org/0000-0002-5935-9777

Deok Hee Kim, https://orcid.org/0000-0002-5617-5067

Young-Woo Kim, https://orcid.org/0000-0002-1559-9672

\section{AUTHORS' CONTRIBUTIONS}

Conceptualization: YWK. Formal analysis: DHK. Methodology: BWE, KWR. Writing-original draft: YWK, WHH. Writing-review and editing: WHH, HMY.

\section{CONFLICT OF INTEREST}

None.

\section{FUNDING}

None.

\section{ACKNOWLEDGMENTS}

WH and YK carried out acquisition of data, statistical analysis, preparation of the manuscript and typing. BE, HY, DK participated in interpretation of data and critical revision. DK. $\mathrm{KR}$, YK participated in study design, interpretation of data and critical revision.

\section{REFERENCES}

1) Kim HH, Hyung WJ, Cho GS, et al. Morbidity and mortality of laparoscopic gastrectomy versus open gastrectomy for gastric cancer: an interim report--a phase III multicenter, prospective, randomized Trial (KLASS Trial). Ann Surg 2010;251:417-420.

2) Kim YW, Baik YH, Yun YH, et al. Improved quality of life outcomes after laparoscopy-assisted distal gastrectomy for early gastric cancer: results of a prospective randomized clinical trial. Ann Surg 2008;248:721-727.

3) Hur H, Xuan Y, Ahn CW, Cho YK, Han SU. Trends and outcomes of minimally invasive surgery for gastric cancer: 750 consecutive cases in seven years at a single center. Am J Surg 2013;205:45-51.

4) Ikeda O, Sakaguchi Y, Aoki Y, et al. Advantages of totally laparoscopic distal gastrectomy over laparoscopically assisted distal gastrectomy for gastric cancer. Surg Endosc 2009;23:2374-2379.

5) Lee HH, Song KY, Lee JS, Park SM, Kim JJ. Delta-shaped anastomosis, a good substitute for conventional Billroth I technique with comparable long-term functional outcome in totally laparoscopic distal gastrectomy. Surg Endosc 2015;29:2545-2552.

6) Wang SY, Hong J, Hao HK. A comparative study of delta-shaped and conventional Billroth I anastomosis after laparoscopic distal gastrectomy for gastric cancer. Surg Endosc 2017;31:3191-3202.

7) Urushihara T, Sumimoto K, Shimokado K, Kuroda Y. Gastric motility after laparoscopically assisted distal gastrectomy, with or without preservation of the pylorus, for early gastric cancer, as assessed by digital dynamic $\mathrm{x}$-ray imaging. Surg Endosc 2004; 18:964-968.

8) Hiki N, Kaminishi M. Pylorus-preserving gastrectomy in gastric cancer surgery--open and laparoscopic approaches. Langenbecks Arch Surg 2005;390:442-447.

9) Horiuchi T, Shimomatsuya T, Chiba Y. Laparoscopically assisted pylorus-preserving gastrectomy. Surg Endosc 2001;15:325-328.

10) Kumagai K, Hiki N, Nunobe S, et al. Totally laparoscopic pylorus- 
preserving gastrectomy for early gastric cancer in the middle stomach: technical report and surgical outcomes. Gastric Cancer 2015;18:183-187.

11) Ohashi M, Hiki N, Ida S, Kumagai K, Nunobe S, Sano T. A novel method of intracorporeal end-to-end gastrogastrostomy in laparoscopic pylorus-preserving gastrectomy for early gastric cancer, including a unique anastomotic technique: piercing the stomach with a linear stapler. Surg Endosc 2018;32:4337-4343.

12) Lee SW, Bouras G, Nomura E, et al. Intracorporeal stapled anastomosis following laparoscopic segmental gastrectomy for gastric cancer: technical report and surgical outcomes. Surg Endosc 2010;24:1774-1780.

13) WHO Expert Consultation. Appropriate body-mass index for Asian populations and its implications for policy and intervention strategies. Lancet 2004;363:157-163.

14) Kim MG, Kim KC, Kim BS, et al. A totally laparoscopic distal gastrectomy can be an effective way of performing laparoscopic gastrectomy in obese patients (body mass index $>/=30$ ). World $\mathrm{J}$ Surg 2011;35:1327-1332.

15) Han WH, Yehuda AB, Kim DH, et al. A comparative study of totally laparoscopic distal gastrectomy versus laparoscopic- assisted distal gastrectomy in gastric cancer patients: Short-term operative outcomes at a high-volume center. Chin J Cancer Res 2018;30:537-545.

16) Chen $\mathrm{K}$, Wu D, Pan Y, et al. Totally laparoscopic gastrectomy using intracorporeally stapler or hand-sewn anastomosis for gastric cancer: a single-center experience of 478 consecutive cases and outcomes. World J Surg Oncol 2016;14:115.

17) Kanaji S, Harada H, Nakayama S, et al. Surgical outcomes in the newly introduced phase of intracorporeal anastomosis following laparoscopic distal gastrectomy is safe and feasible compared with established procedures of extracorporeal anastomosis. Surg Endosc 2014;28:1250-1255.

18) Jeong O, Jung MR, Park YK, Ryu SY. Safety and feasibility during the initial learning process of intracorporeal Billroth I (deltashaped) anastomosis for laparoscopic distal gastrectomy. Surg Endosc 2015;29:1522-1529.

19) Park KB, Kwon OK, Yu W, Jang BC. Body composition changes after totally laparoscopic distal gastrectomy with delta-shaped anastomosis: a comparison with conventional Billroth I anastomosis. Surg Endosc 2016;30:4286-4293. 